\title{
VALUE OF SERUM ALPHA L FUCOSIDASE LEVEL AS A PROGNOSTIC BIOMARKER FOR HEPATOCELLULAR CARCINOMA BEFORE AND AFTER CHEMO-EMBOLIZATION AND RADIO-FREQUENCY, A PROSPECTIVE STUDY

\author{
By
}

AHMED SAMIR ABO HALIMA*, MARCEL WILLIAM KEDDEAS, And HANY HAROUN KAISAR*

Department of Gastroenterology and Hepatology, Faculty of Medicine, Ain Shams

University, Cairo 11211, Egypt ( ${ }^{*}$ Correspondence: dr.abohalima@hotmail.com

dr_hanyharoun@yahoo.com, Mobile + 20122368 0203)

Abstract

Because hepatocellular carcinoma (HCC) is a complex disease with multiple risk pathogenic mechanisms due to misdiagnosis with a single biomarker. A combination of biomarkers may be more valuable for the diagnosis, staging and prognosis of HCC. In the near future, identifying non-invasive and cost-effective biomarkers for early diagnosis and personalized treatment of HCC will be one of the most promising fields of biomarker research. This study assessed the alpha $\mathrm{L}$ fucosidase (AFU) value as a prognostic biomarker in patients with HCC before and after chemo-embolization and radio-frequency. A total of 60 subjects were subdivided into 3 groups: GI: 30 HCC patients underwent interventional management (chemo-embolization or radiofrequency), GII: 20 liver cirrhosis (LC) patients and GIII: 10 cross-matched individuals as control

The results showed that plasma AFU had significantly higher diagnostic performance in HCC diagnosis than alpha fetoprotein (AFP) at a cut off value of $>2.5 \mathrm{u} / 1$, with sensitivity $100 \%$, specificity $95 \%$, positive predictive value (PPV) $96.8 \%$, negative predictive value (NPV) $100 \%$ and diagnostic accuracy $98 \%$. Basal pre-intervention AFU) had significantly high diagnostic performance to predict $\mathrm{HCC}$ recurrence after intervention at a cut off value of $>12.5 \mathrm{u} / \mathrm{l}$ with sensitivity $100 \%$, specificity $92 \%$, PPV, $71.4 \%$, NPV $100 \%$ and diagnostic accuracy $93.3 \%$. Postintervention AFU had significant moderate diagnostic performance in predicting recurrence of $\mathrm{HCC}$ at cut off value of $>7.5 \mathrm{u} / 1$ with sensitivity $80 \%$, specificity $92 \%$, positive predictive value (PPV) 66.7\%, negative predictive value (NPV) $95.8 \%$ \& diagnostic accuracy $90 \%$.

Keywords: Egypt, Patients, Alpha L Fucosidase, Alphafetoprotein, HCC, Chemo-embolization.

\section{Introduction}

In Egypt, hepatocellular carcinoma (HCC) is usually detected in an advanced stage at which no treatment may be effective including surgery. Early detection of the disease is thus an important goal allowing the patient to be treated before its metastasis to distant organs. Alpha fetoprotein (AFP) which is the golden marker for HCC is of low sensitivity (Zhao et al, 2013), but not secreted in all hepatocellular carcinoma (HCC) and may be normal in as many as $40 \%$ of patients with early HCC (Ibrahim et al, 2013).

Lots of tumor biomarkers were conducted as a complement or substitute for AFP in order to improve sensitivity and specificity in diagnosing HCC (Faria et al, 2019). Additional markers such as alpha $\mathrm{L}$ fucosidase (AFU), transforming growth factors alpha and beta (TGF- $\alpha$ \& TGF- $\beta$ ) and interleukin8 (IL-8) detected HCC (Saad et al, 2020).

Fucosylation of glycoproteins (the addition of L-fucose at the terminal end of the oligosaccharide chain) is one of the most important features that mediate several specific biologic functions. It has been documented that tumor cells modulate their surface by increasing fucosylation levels to escape recognition, which contribute to several abnormal characteristics of tumor cells, such as decreased adhesion and uncontrolled tumor growth. Alpha L fucosidase (AFU) proved as useful tumor marker for HCC in French population (Waidely et al, 2017)

Alpha L fucosidase (AFU) is a lysosomal glycosidase found in all mammalian cells concerned with the degradation of a variety of the fucose-containing glyco-conjugates. 
Higher activities of the enzyme were detected in HCC patients. Furthermore, the persistently elevated AFU level in patients with cirrhosis adds to the detection of HCC at an earlier stage owing to elevated activity of AFU at least 6 months before the detection of HCC by ultrasonography in $85 \%$ of patients. AFU is present in minute concentrations in all animal tissues but was found to be overexpressed in cancerous tissue, particularly with HCC. Purified AFU and corresponding polyclonal antibody can be used as antigen-antibody candidates to detect primary HCC at early stage (Darnell et al, 2015).

The study aimed to assess the value of alpha L fucosidase (AFU) as a prognostic biomarker in patients with HCC before and after chemo-embolization and radio-frequency.

\section{Patients and Methods}

A total of 60 subjects selected from outpatient and inpatient Hepatology Department at Ain Shams University Hospital during the period from October 2016 to October 2018. They were subdivided into 3 groups; GI (HCC) included 30 patients with HCC diagnosed by triphasic pelviabdominal CT imaging showing early arterial uptake followed by rapid washout in the venous phase which is highly specific for HCC (Reig et al, 2014), GII (LC) included 20 patients with liver cirrhosis (LC) diagnosed by laboratory Investigations and abdominal ultrasonography and GIII (controls) included 10 crossmatched healthy subjects.

Exclusion criteria in this study were patients with liver metastasis, patients with advanced stage (C) or terminal stages (D) according to Barcelona Clinic Liver Cancer (BCLC) staging system (6), patients with suspected colorectal cancers (high level of carcinoembryonic antigen (CEA), patients with suspected pancreatic cancer (high level of cancer antigen 19.9 (CA 19.9), female patients with suspected ovarian malignancy (high level of cancer antigen 125 (CA 125). All participants agreed to the study conditions and provided a written informed consent before being enrolled.
All participants were subjected to complete history taking, clinical examination, pelviabdominal ultrasound and laboratory investigations that included total leucocytic count (TLC), Hb concentration, platelet count (PLT), serum creatinine, liver function tests (AST, ALT, ALP, albumin), total bilirubin, direct bilirubin, INR, hepatitis markers (HCVAb, HBsAg), tumor markers (CEA, CA 125, CA 19.9), serum alpha fetoprotein (AFP) and serum alpha L fucosidase (AFU). Child-Pugh score was done for all patients Serum alpha fetoprotein was measured by human AFP EIA kit lot. REF 600-10 manufactured by CanAg Diagnostics AB, Majnabble Terminal SE-414 55 Gothenburg, and Sweden (Siegel et al, 2013).

Serum alpha L fucosidase enzyme was measured by ELISA using Human alpha-Lfucosidase (AFU) ELISA Kit by Glory Science Co., Del Rio, USA. Blood samples were taken from patients in complete aseptic condition, centrifuged at the speed of 2000$3000 \mathrm{rpm}$ for $20 \mathrm{~min}$ then supernatant was removed. The samples were kept in $-20^{\circ} \mathrm{C}$. Kit was for the quantitative level of AFU in the sample, adopt purified human AFU to coat microliter plate, made solid phase antibody, then added samples or standards to wells with a labeled antibody specific to AFU, then add labeled Horseradish peroxidase (HRP) to the well. After washing completely, add Tetramethylbenzidine (TMB) substrate solution. TMB substrate becomes blue color in wells that contains antibodyantigen-enzyme-antibody complex. Reaction was terminated by a stop solution and color change was measured at $450 \mathrm{~nm}$ wavelength. Concentration of AFU in samples was then determined by comparing the optical density (OD) of the samples to the standard curve. Calculation of the results was done by taking the standard concentration as the horizontal, the OD value for vertical, draw the standard curve on graph paper. Find out corresponding concentration according to the sample OD value by the sample curve multiplied by the dilution multiple, or calculate the straight 
line regression equation of standard curve with concentration and OD value, with the sample equation, calculate the sample concconcentration, multiplied by dilution factor

Triphasic pelviabdomenal CT was done to patients with HCC. The portal vein patency, number and overall size of HCC were detected with characteristic rapid arterial uptake and early washout in venous phase. Radiological intervention was selected according to BCLC staging system. Patients who were very early \& early-stage HCC (BCLC 0 or BCLC A) who had a solitary lesion or up to 3 nodules $<3 \mathrm{~cm}$ (without macrovascular invasion or extrahepatic spread) with preserved liver function underwent radiofrequency ablation (RF). Asymptomatic patients with intermediate-stage HCC (BCLC B) had large and/or multifocal tumors without vascular invasion or spread beyond liver with preserved liver function underwent trans arterial chemoembolization (TACE).

At Radiology Department did interventional management. Serum alpha fetoprotein (AFP), serum alpha L fucosidase (AFU) and triphasic pelviabdominal CT were repeated 4 weeks after intervention.

Statistical analysis: Data were tabulated, and analyzed (IBM SPSS, version 22.0, Chicago, USA, 2013. Descriptive statistics were done for quantitative data as minimum \& maximum range and mean $\pm \mathrm{SD}$ (standard deviation) for quantitative normally distributed data, median and $1^{\text {st }} \& 3^{\text {rd }}$ inter-quartile range for quantitative non-normally distributed data while it was done for qualitative data as number $\&$ percentage. Analysis was done for quantitative variables using independent t-test in cases of two independent groups with normally distributed data, Mann whiteny $U$ test in cases of two independent groups with non-normally distributed data, Wilcoxon signed rank test in cases of 2 dependent groups with non-parametric data. ANOVA test was done for more than 2 independent groups with normally distributed data and Kruskal Wallis test with post hoc Dunn's test for more than 2 independent groups without normal distributed data.

ROC curve evaluated performance of different tests to differentiate between groups. Probability of error $(p)$ was expressed as: $\mathrm{p}$ value $\geq 0.05$ : non-significant, $p$ value $\leq$ to 0.05 : significant and $\mathrm{p}$ value less than 0.01 : highly significant.

Ethics approval: All procedures performed were approved by Ain Shams University Ethical Research Committee and went with the 1964 Helsinki declaration \& later amendments. Approval no. 000017585 . A written consent was obtained from all participants.

\section{Results}

The study was carried on 60 subjects selected from outpatient and inpatient Hepatology Department, Ain Shams University Hospitals from October 2016 to October 2018. They were subdivided into 3 groups: GI (HCC) 30 patients who underwent interventional management in radiology department, GII (LC) 20 patients with liver cirrhosis and GIII (Control) 10 healthy subjects with ages ranged between $41 \& 69$ years.

HCC patients were $23(76.6 \%)$ males and $7(23.4 \%)$ females, LC patients were 17 $(85 \%)$ males and $3(15 \%)$ females, and controls were $8(80 \%)$ males and $2(20 \%)$ females, without significant difference as to demographic characteristics. All patients $\mathrm{HCC}$ \& LC were HCVAb positive and HBsAg negative. HCC patients had significant lower child score than LC ones due to BCLC inclusion criteria. In HCC 28(93.3\%) underwent TACE and 2(6.7\%) patients underwent RF.

Laboratory findings of patients showed hemoglobin concentration, platelet count and serum albumin were statistically higher among HCC patients than LC patients. Serum AST, ALP, total bilirubin, direct bilirubin and INR were significantly higher among LC than HCC patients, but without significant different as to TLC, serum ALT \& creatinine.

Before radiological intervention, both serum AFP and AFU were highest in $\mathrm{HCC}$ patients with highly significant difference $(p<0.0001)$ between HCC, LC and controls. 
In LC both markers were highly significant $(p<0.0001)$ as compared with control, but significant low as compared with HCC.

At a cut off value of $>6.0 \mathrm{ng} / \mathrm{mL}$, basal (before intervention) AFP showed significantly moderate diagnostic performance with sensitivity $93.3 \%$, specificity $70 \%$, PPV $82.4 \%$, NPV $87.5 \%$ \& diagnostic accuracy $84 \%$. At cut off value of $>2.5 \mathrm{u} / \mathrm{l}$, basal (before intervention) AFU showed significantly high diagnostic performance in differentiating $\mathrm{HCC}$ from liver cirrhosis with sensitivity $100 \%$, specificity 95\%, PPV 96.8\%, NPV 100\% \& diagnostic accuracy $98 \%$.

After radiological intervention, AFP \& AFU significantly decreased, 4 weeks post radiological intervention, recurrence occurred in 5(16.6\%) HCC, of whom 4(13.3\%) underwent TACE and one (3.3\%) RF.

Serum AFP among recurrent cases was not high significantly before and after intervention, without significant reduction in serum AFP among recurrent \& non-recurrent patients. Serum AFU of recurrent cases was significantly higher before \& after intervention, with significant reduction in AFU in recurrent \& non-recurrent patients. At cut off value of $>12.5 \mathrm{u} / 1$ basal (before intervention) AFU showed significantly high diagnostic performance in predicting HCC recurrence with sensitivity $100 \%$, specificity 92\%, PPV 71.4\%, NPV 100\% \& diagnostic accuracy $93.3 \%$. At cut off value of $>7.5 \mathrm{u} / 1$, serum AFU after intervention had significantly moderate diagnostic performance in detecting HCC patients recurrence with sensitivity $80 \%$, specificity $92 \%$, PPV $66.7 \%$, NPV $95.8 \%$ \& diagnostic accuracy $90 \%$.

Details were given in tables $(1,2,3,4,5$, $6,7,8,9,10,11 \& 12) \&$ in figures $(1 \& 2)$

Table 1: Comparison between HCC and LC groups as regards child score, grade \& HCC intervention

\begin{tabular}{|c|c|c|c|c|c|}
\hline \multicolumn{2}{|c|}{ Variables } & $\mathrm{HCC}(\mathrm{N}=30)$ & LC $(\mathrm{N}=20)$ & Test value & $\mathrm{P}$ \\
\hline \multirow{2}{*}{$\begin{array}{l}\text { Child } \\
\text { score }\end{array}$} & $\mathrm{M} \pm \mathrm{SD}$ & $6.0 \pm 1.0$ & $8.1 \pm 1.4$ & \multirow[t]{2}{*}{$\mathrm{t}: 6.20$} & \multirow{2}{*}{$<0.001 *$} \\
\hline & Range & $5.0-8.0$ & $6.0-11.0$ & & \\
\hline \multirow{3}{*}{$\begin{array}{l}\text { Child } \\
\text { grade } \\
(\mathrm{n}, \%)\end{array}$} & A & $23(76.7 \%)$ & $2(10.5 \%)$ & \multirow{3}{*}{ E: 22.13} & \multirow{3}{*}{$<0.001 *$} \\
\hline & $\mathrm{B}$ & $7(23.3 \%)$ & $14(73.7 \%)$ & & \\
\hline & $\mathrm{C}$ & $0(0.0 \%)$ & $3(15.8 \%)$ & & \\
\hline \multirow{2}{*}{$\begin{array}{l}\text { Inter- } \\
\text { vention }\end{array}$} & TACE & $28(93.3 \%)$ & -- & \multirow[b]{2}{*}{--} & \multirow[b]{2}{*}{--} \\
\hline & $\mathrm{RF}$ & $2(6.7 \%)$ & -- & & \\
\hline
\end{tabular}

Table 2: Basal (before intervention) levels of AFP and AFU among groups

\begin{tabular}{|c|c|c|c|c|c|c|}
\hline \multicolumn{2}{|c|}{ Variables } & $\mathrm{HCC}(\mathrm{N}=30)$ & $\mathrm{LC}(\mathrm{N}=20)$ & Control(N=10) & T-value & ${ }^{\wedge} \mathrm{P}$ \\
\hline \multirow{3}{*}{$\begin{array}{c}\mathrm{AFP} \\
(\mathrm{ng} / \mathrm{mL})\end{array}$} & Median (IQR) & $12.0(8.6-67.8)$ & $4.5(4.2-6.7)$ & $3.8(3.0-4.0)$ & \multirow{3}{*}{$\begin{array}{c}\chi^{2} \\
29.58\end{array}$} & \multirow{3}{*}{$<0.001 *$} \\
\hline & Range & $3.7-454.2$ & $3.1-119.8$ & $2.0-4.6$ & & \\
\hline & $\mathrm{HG}$ & $\mathrm{a}$ & $\mathrm{b}$ & $\mathrm{c}$ & & \\
\hline \multirow{3}{*}{$\begin{array}{l}\text { AFU } \\
\text { (U/L) }\end{array}$} & Median (IQR) & $8.5(3.9-11.9)$ & $1.5(1.1-2.0)$ & $0.4(0.2-0.5)$ & \multirow{3}{*}{$\begin{array}{c}\chi^{2} \\
47.95\end{array}$} & \multirow{3}{*}{$<0.001^{*}$} \\
\hline & Range & $2.5-15.0$ & $0.9-5.0$ & $0.1-0.6$ & & \\
\hline & $\mathrm{HG}$ & $\mathrm{a}$ & $\mathrm{b}$ & $\mathrm{C}$ & & \\
\hline
\end{tabular}

Table 3: Diagnostic performance of basal AFU and AFP in differentiating HCC from LC groups

\begin{tabular}{|c|c|c|c|c|c|}
\hline Marker & AUC & SE & P & $95 \%$ CI & Cut off \\
\hline Basal AFP & 0.815 & 0.066 & $<0.001 *$ & $0.685-0.945$ & $\geq 6.0$ \\
\hline Basal AFU & 0.987 & 0.014 & $<0.001 *$ & $0.500-1.000$ & $\geq 2.5$ \\
\hline
\end{tabular}

Table 4: Diagnostic characteristics of AFU \& AFP in differentiating HCC from LC

\begin{tabular}{|c|c|c|c|c|}
\hline \multirow{2}{*}{ Characters } & \multicolumn{2}{|c|}{$\mathrm{AFU} \geq 2.5 \mathrm{U} / \mathrm{L}$} & \multicolumn{2}{c|}{$\mathrm{AFP} \geq 6.0 \mathrm{ng} / \mathrm{mL}$} \\
\cline { 2 - 5 } & Value & $95 \% \mathrm{CI}$ & Value & $95 \% \mathrm{CI}$ \\
\hline Sensitivity & $100.0 \%$ & $88.4 \%-100.0 \%$ & $93.3 \%$ & $77.9 \%-99.2 \%$ \\
\hline Specificity & $95.0 \%$ & $75.1 \%-99.9 \%$ & $70.0 \%$ & $45.7 \%-88.1 \%$ \\
\hline DA & $98.0 \%$ & $89.4 \%-99.9 \%$ & $84.0 \%$ & $70.9 \%-92.8 \%$ \\
\hline YI & $95.0 \%$ & $85.4 \%-104.6 \%$ & $63.3 \%$ & $41.4 \%-85.3 \%$ \\
\hline PPV & $96.8 \%$ & $83.3 \%-99.9 \%$ & $82.4 \%$ & $65.5 \%-93.2 \%$ \\
\hline NPV & $100.0 \%$ & $82.4 \%-100.0 \%$ & $87.5 \%$ & $61.7 \%-98.4 \%$ \\
\hline LR+ & 20.00 & $2.961-135.107$ & 3.11 & $1.582-6.118$ \\
\hline LR- & 0.00 & $0.00-0.00$ & 10.50 & $2.670-41.292$ \\
\hline LR & $>100.0$ & $>100.0->100.0$ & 32.67 & $5.825-183.179$ \\
\hline Kappa & 0.96 & $0.877-1.039$ & 0.66 & $0.440-0.870$ \\
\hline
\end{tabular}


Table 5: Laboratory data among HCC \& LC groups

\begin{tabular}{|c|c|c|c|c|c|}
\hline \multicolumn{2}{|c|}{ Variables } & $\mathrm{HCC}(\mathrm{N}=30)$ & $\mathrm{LC}(\mathrm{N}=20)$ & Test value & ${ }^{\wedge} \mathrm{P}$ \\
\hline \multirow{2}{*}{$\begin{array}{l}\mathrm{Hb} \\
(\mathrm{gm} / \mathrm{dL})\end{array}$} & $\mathrm{M} \pm \mathrm{SD}$ & $12.6 \pm 1.2$ & $11.1 \pm 1.7$ & \multirow{2}{*}{$\mathrm{t}: 3.42$} & \multirow{2}{*}{$0.002 *$} \\
\hline & Range & $10.5-15.0$ & $8.3-13.8$ & & \\
\hline \multirow{2}{*}{$\begin{array}{l}\text { TLC } \\
\left(\mathrm{x} 10^{3} / \mathrm{mL}\right)\end{array}$} & $\mathrm{M} \pm \mathrm{SD}$ & $5.8 \pm 1.4$ & $5.0 \pm 1.8$ & \multirow{2}{*}{$\mathrm{t}: 1.70$} & \multirow{2}{*}{0.097} \\
\hline & Range & $4.0-9.5$ & $1.4-9.4$ & & \\
\hline \multirow{2}{*}{$\begin{array}{l}\text { PLT } \\
\left(\mathrm{x} 10^{3} / \mathrm{mL}\right)\end{array}$} & $\mathrm{M} \pm \mathrm{SD}$ & $116.0 \pm 47.3$ & $68.9 \pm 32.0$ & \multirow{2}{*}{$\mathrm{t}: 3.89$} & \multirow{2}{*}{$<0.001^{*}$} \\
\hline & Range & $65.0-265.0$ & $24.0-123.0$ & & \\
\hline \multirow{2}{*}{$\begin{array}{l}\text { ALT } \\
(\mathrm{IU} / \mathrm{L})\end{array}$} & $\mathrm{M} \pm \mathrm{SD}$ & $39.8 \pm 30.9$ & $36.1 \pm 11.0$ & \multirow{2}{*}{$\mathrm{t}: 0.51$} & \multirow{2}{*}{0.611} \\
\hline & Range & $11.0-185.0$ & $17.0-57.0$ & & \\
\hline \multirow{2}{*}{$\begin{array}{l}\text { AST } \\
(\mathrm{IU} / \mathrm{L}) \\
\end{array}$} & $\mathrm{M} \pm \mathrm{SD}$ & $38.7 \pm 24.8$ & $57.7 \pm 18.1$ & \multirow{2}{*}{$\mathrm{t}:-2.93$} & \multirow{2}{*}{$0.005^{*}$} \\
\hline & Range & $16.0-132.0$ & $25.0-85.0$ & & \\
\hline \multirow{2}{*}{$\begin{array}{l}\text { ALP } \\
(\mathrm{IU} / \mathrm{L})\end{array}$} & $\mathrm{M} \pm \mathrm{SD}$ & $78.4 \pm 22.2$ & $115.1 \pm 49.8$ & \multirow{2}{*}{$\mathrm{t}:-3.09$} & \multirow{2}{*}{$0.005 *$} \\
\hline & Range & $35.0-140.0$ & $34.0-232.0$ & & \\
\hline \multirow{2}{*}{$\begin{array}{l}\text { Albumin } \\
\text { (g/dL) }\end{array}$} & $\mathrm{M} \pm \mathrm{SD}$ & $3.8 \pm 0.4$ & $2.3 \pm 0.5$ & \multirow{2}{*}{$\mathrm{t}: 12.51$} & \multirow{2}{*}{$<0.001 *$} \\
\hline & Range & $2.9-4.5$ & $1.3-3.2$ & & \\
\hline \multirow{2}{*}{$\begin{array}{l}\text { Total bilirubin } \\
(\mathrm{mg} / \mathrm{dL})\end{array}$} & $\mathrm{M} \pm \mathrm{SD}$ & $0.9 \pm 0.3$ & $3.1 \pm 1.6$ & \multirow{2}{*}{$\mathrm{t}:-6.22$} & \multirow{2}{*}{$<0.001^{*}$} \\
\hline & Range & $0.4-1.7$ & $1.3-6.3$ & & \\
\hline \multirow{2}{*}{$\begin{array}{l}\text { Direct bilirubin } \\
(\mathrm{mg} / \mathrm{dL})\end{array}$} & $\mathrm{M} \pm \mathrm{SD}$ & $0.5 \pm 0.2$ & $1.4 \pm 0.8$ & \multirow{2}{*}{$\mathrm{t}:-4.74$} & \multirow{2}{*}{$<0.001 *$} \\
\hline & Range & $0.1-1.1$ & $0.3-3.0$ & & \\
\hline \multirow{2}{*}{ INR } & $\mathrm{M} \pm \mathrm{SD}$ & $1.21 \pm 0.17$ & $1.59 \pm 0.27$ & \multirow{2}{*}{$\mathrm{t}:-6.16$} & \multirow{2}{*}{$<0.001^{*}$} \\
\hline & Range & $0.70-1.70$ & $1.20-2.30$ & & \\
\hline \multirow{2}{*}{$\begin{array}{l}\text { Creatinine } \\
(\mathrm{mg} / \mathrm{dL})\end{array}$} & $\mathrm{M} \pm \mathrm{SD}$ & $0.90 \pm 0.16$ & $1.02 \pm 0.33$ & \multirow{2}{*}{$\mathrm{t}:-1.50$} & \multirow{2}{*}{0.148} \\
\hline & Range & $0.60-1.20$ & $0.70-2.00$ & & \\
\hline
\end{tabular}

${ }^{\wedge}$ Independent t-test, *Significant, ^ANOVA, *Significant, HG: (with same letter by post hoc Bonferroni test)

Table 6: Serum AFP among HCC group before and after intervention

\begin{tabular}{|c|c|c|c|c|}
\hline Variables & Median (IQR) & Range & Test value & $\mathrm{P}$ \\
\hline Before & $12.0(8.6-67.8)$ & $3.7-454.2$ & \multirow{2}{*}{ Z: } & \multirow{2}{*}{$<0.001^{*}$} \\
\hline After & $11.5(5.0-44.0)$ & $1.4-198.0$ & \multirow{2}{*}{3.74} & \\
\hline \#Reduction & $4.2(0.4-10.3)$ & $-5.0-256.2$ & & \\
\hline
\end{tabular}

\#Negative = elevation, IQR: Interquartile range, ${ }^{\wedge}$ Wilcoxon signed rank test (z value), ${ }^{*}$ Significant Table 7: Serum AFU among HCC patients before and after intervention

\begin{tabular}{|c|c|c|c|c|}
\hline Variables & Median (IQR) & Range & Test value & P \\
\hline Before & $8.5(3.9-11.9)$ & $2.5-15.0$ & & \multirow{2}{*}{ z: 4.71} \\
\hline After & $2.8(2.0-6.0)$ & $1.0-11.0$ & & \\
\cline { 1 - 3 } Reduction & $3.7(2.0-6.1)$ & $0.0-11.0$ & & \\
\hline
\end{tabular}

\#Negative values indicate elevation, IQR: Interquartile range, ${ }^{\wedge}$ Wilcoxon signed rank test (z value), ${ }^{*}$ Significant Table 8: Failure of treatment among HCC patients

\begin{tabular}{|c|c|c|c|}
\hline Recurrence & Intervention & No. & $\%$ \\
\hline \multirow{2}{*}{ Present } & TACE & 4 & \multirow{2}{*}{16.6} \\
\cline { 2 - 3 } & RF & 1 & \\
\hline \multirow{2}{*}{ Absent } & TACE & 24 & \multirow{2}{*}{83.3} \\
\cline { 2 - 3 } & RF & 1 & \\
\hline
\end{tabular}

Table 9: Comparison between recurrent $\&$ non-recurrent cases as toserum AFP levels (Basal $\&$ after intervention)

\begin{tabular}{|c|c|c|c|c|}
\hline Variables & Recurrent $(\mathrm{N}=5)$ & Non-recurrent $(\mathrm{N}=25)$ & Test value & $\wedge \mathrm{P}$ \\
\hline Basal AFP $(\mathrm{ng} / \mathrm{mL})$ & $97.0(8.1-140.5)$ & $11.5(8.3-38.4)$ & $\mathrm{Z}:-0.95$ & 0.344 \\
\hline After AFP $(\mathrm{ng} / \mathrm{mL})$ & $29.3(5.6-79.1)$ & $11.0(4.7-41.0)$ & $\mathrm{Z}:-0.81$ & 0.448 \\
\hline Reduction AFP $(\mathrm{ng} / \mathrm{mL})$ & $25.3(2.5-82.7)$ & $3.9(3.0-6.0)$ & $\mathrm{Z}:-1.64$ & 0.108 \\
\hline Test value & -1.753 & -0.272 & & \\
\hline \#P & 0.080 & 1.000 & & -- \\
\hline
\end{tabular}

${ }^{\wedge}$ Mann Whitney test (z value), \#Wicoxon signed rank test (z value) (Comparison between before \& after), *Significant

Table 10: Comparison between recurrent and non-recurrent cases regarding serum AFU levels (Basal \& after intervention)

\begin{tabular}{|c|c|c|c|c|}
\hline Variables & Recurrent $(\mathrm{N}=5)$ & Non-recurrent $(\mathrm{N}=25)$ & Test value & $\mathrm{P}$ \\
\hline Basal AFU (U/L) & $14.0(13.0-15.0)$ & $7.0(3.6-9.5)$ & z: -3.21 & $<0.001^{*}$ \\
\hline After AFU (U/L) & $9.0(5.8-10.1)$ & $2.0(1.8-5.0)$ & z: -2.72 & $0.006^{*}$ \\
\hline Reduction AFU (U/L) & $6.0(2.9-8.7)$ & $3.0(1.8-6.0)$ & z: -1.23 & 0.219 \\
\hline Test value & -2.023 & -4.290 & & \\
\hline \#P & $0.043^{*}$ & $<0.001^{*}$ & & -- \\
\hline
\end{tabular}


Table 11: Diagnostic performance of serum AFU in prediction of HCC recurrence

\begin{tabular}{|c|c|c|c|c|c|c|}
\hline Marker & AUC & SE & P & C5\% CI & Cut off \\
\hline Basal AFU & 0.960 & 0.035 & & $<0.001 *$ & $0.500-1.000$ & $\geq 12.5$ \\
\hline After AFU & 0.888 & 0.074 & & 0.007 & $0.500-1.000$ & $\geq 7.5$ \\
\hline Reduction AFU & 0.676 & 0.122 & 0.221 & $0.500-0.916$ & -- \\
\hline
\end{tabular}

Table 12: Diagnostic characteristics of AFU and AFP in prediction of $\mathrm{HCC}$ recurrence

\begin{tabular}{|c|c|c|c|c|}
\hline \multirow{2}{*}{ Characters } & \multicolumn{2}{|c|}{ Basal AFU $\geq 12.5$ (Prognostic) } & \multicolumn{2}{c|}{ After AFU $\geq 7.5$ (Diagnostic) } \\
\cline { 2 - 5 } & Value & $95 \%$ CI & Value & $95 \%$ CI \\
\hline Sensitivity & $100.0 \%$ & $47.8 \%-100.0 \%$ & $80.0 \%$ & $28.4 \%-99.5 \%$ \\
\hline Specificity & $92.0 \%$ & $74.0 \%-99.0 \%$ & $92.0 \%$ & $74.0 \%-99.0 \%$ \\
\hline DA & $93.3 \%$ & $77.9 \%-99.2 \%$ & $90.0 \%$ & $73.5 \%-97.9 \%$ \\
\hline Youden's index & $92.0 \%$ & $81.4 \%-102.6 \%$ & $72.0 \%$ & $35.4 \%-108.6 \%$ \\
\hline PPV & $71.4 \%$ & $29.0 \%-96.3 \%$ & $66.7 \%$ & $22.3 \%-95.7 \%$ \\
\hline NPV & $100.0 \%$ & $85.2 \%-100.0 \%$ & $95.8 \%$ & $78.9 \%-99.9 \%$ \\
\hline LR+ & 12.50 & $3.308-47.231$ & 10.00 & $2.467-40.540$ \\
\hline LR- & 0.00 & $0.00-0.00$ & 4.60 & $0.794-26.653$ \\
\hline LR & $>100.0$ & $>100.0->100.0$ & 46.00 & $3.333-634.883$ \\
\hline Kappa & 0.79 & $0.522-1.064$ & 0.67 & $0.319-1.014$ \\
\hline
\end{tabular}

CI: Confidence interval, YI: Youden's index, DA: Diagnostic accuracy, PPV: Positive Predictive value, NPV: Negative Predictive value, LR+: Positive likelihood ratio, LR-: Negative likelihood ratio, LR: Diagnostic odd ratio.

\section{Discussion}

Despite progress made during the past few decades, HCC is still one of the most frequent and deadly cancers worldwide particularly in Egypt (Abdel-Bary et al, 2012). Globally, there were 750,000 new cases of annually liver cancer about $70-85 \%$ of which were HCC. Due to the asymptomatic nature of an early HCC case and lack of effective diagnostic and screening strategies, most patients $(>80 \%)$ were with HCC advanced stage, with poor prognosis. So, early detection of HCC is a significant public health issue. Tumor biomarker was effective to screen $\mathrm{HCC}$ as, non-invasive, inexpensive with a high accuracy (Negahdary et al, 2015).

AFP is a golden marker for $\mathrm{HCC}$, but with low sensitivity (Choi et al, 2013). Many tumor biomarkers were conducted to substitute AFP to improve sensitivity and specificity in diagnosing HCC included embryonic antigens, proteantigens, enzymes, isoenzymes, cytokines, growth factors and molecular biomarkers (Wang and Cao, 2004).

The present study evaluated the serum alpha $\mathrm{L}$ fucosidase level (AFU) as a diagnostic and prognostic biomarker for HCC before and after radiological intervention as compared with serum AFP. This study included 60 subjects divided into three groups; 30 patients with HCC, 20 with liver cirrhosis (LC) and 10 normal subjects as control. Radiolog- ical intervention was done to HCC. Serum levels of AFP and AFU were measured before and after intervention.

While measuring AFP, it was highest in $\mathrm{HCC}$ with median $(12.0 \mathrm{ng} / \mathrm{ml})$ followed by liver cirrhotic group with median $(4.5 \mathrm{ng} / \mathrm{ml})$ and least in control with median $(3.8 \mathrm{ng} / \mathrm{ml})$ and the elevation of AFP was statistically highly significant in all groups. This agreed with Montaser et al. (2012) who found that elevation in median serum AFP in as compared with liver cirrhosis $(11.1 \mathrm{ng} / \mathrm{ml})$ and control $(2.03 \mathrm{ng} / \mathrm{ml})$. But, Mossad et al. (2014) found that serum AFP was elevated in HCC as compared to liver cirrhosis group, but without significant.

In the present study, AFU was higher in HCC with median (8.5U/L) followed by liver cirrhotic ones with median $(1.5 \mathrm{U} / \mathrm{L})$ and least in controls with median $(0.4 \mathrm{U} / \mathrm{L})$ and the elevation of AFU was highly significant in all groups. This significant difference implied the diagnostic role of AFU in detection of $\mathrm{HCC}$ in cirrhotic patients. Also, agreed with Montaser et al. (2012) who found elevation in median serum AFU level in $\mathrm{HCC}$ $(9.28 \mu \mathrm{mol} / \mathrm{L} / \mathrm{min})$ was highly significant as compared with liver cirrhosis patient $(0.9$ $\mu \mathrm{mol} / \mathrm{L} / \mathrm{min}) \&$ controls $(0.42 \mu \mathrm{mol} / \mathrm{L} / \mathrm{min})$. Wang and Cao (2004) found that mean value of serum AFU activity in patients with HCC was significantly higher than those with cir- 
rhosis $(p<0.01)$, chronic hepatitis $(p<0.01)$, other malignant neoplasm $(p<0.01)$, other diseases $(p<0.01)$ and controls $(p<0.01)$, without significant difference between controls and patients with cirrhosis, chronic hepatitis, other malignant neoplasm or other diseases. El-Tayeh et al. (2012) found that HCC patients showed highest (AFU) enzyme activity, but without significant difference from controls.

Based on significant increase of markers $\mathrm{AFU}$ and AFP in $\mathrm{HCC}$ rather than in liver cirrhosis and control, ROC curves calculated sensitivity and specificity of AFU and AFP. Optimal cut off values selected by ROC curves were $(6.0 \mathrm{ng} / \mathrm{ml})$ for AFP and $(2.5$ $\mathrm{U} / \mathrm{L})$ for AFU, which had significant higher diagnostic performance than AFP in HCC from liver cirrhosis in patients with chronic liver disease. Also, the present results agreed with Montaser et al. (2012) who found that serum AFU level at cut off $(2.3 \mu \mathrm{mol} / \mathrm{L} / \mathrm{min})$, sensitivity was $90 \%$, specificity was $97 \%$ with PPV of $97.2 \%$, NPV of $92.9 \%$ with diagnostic accuracy of $94.9 \%$. Gan et al. (2013) reported that AFU showed higher sensitivity, specificity \& overall accuracy than AFP in HCC in cirrhotic patients \& AFU pooled sensitivity more than AFP (0.72 vs. 0.61$)$.

In the present study, there was no significant correlation between AFU \& AFP in all groups. This agreed with Takahashi et al. (1994), Malaguarnera et al. (2010) and Mossad et al. (2014), who didn't find correlation between serum AFU \& AFP.

In the present study, there was no significant decrease in AFP serum levels after successful radiological intervention for HCC. This agreed with Adaninggar et al. (2016) who didn't find significant difference between serum level of AFP before and one month later. But, Montaser et al. (2012) who found significant decrease in serum AFP after successful intervention.

The present study showed significant reduction in AFU serum level after a successful HCC intervention. This agreed with Wang and Cao (2004) they found that AFU activity in HCC with significant decrease post chemo-therapy or operation within a week to a month. AFU activity dropped to normal. Serum AFU activity correlated with curative effect with good value in post-treatment of HCC efficacy patients (Zhao et al, 2013)

\section{Conclusion}

The outcome results showed that serum AFU levels were significantly higher in HCC patients as compared to liver cirrhosis or controls. Serum AFU has higher sensitivity \& specificity than AFP in HCC diagnosis. Serum AFU dropped after radiological intervention for HCC and to monitor response to therapy. AFU had a potential value as a diagnostic as well as a prognostic marker of HCC to assess the efficacy of any intervention used to treat the disease.

Authors Contributions: All authors equally contributed in this study

Conflict of interest: Authors neither have conflict of interest nor received funds.

\section{Acknowledgements}

The authors express their gratitude to the staff members, Department of Hepatology, Ain Shams Faculty of Medicine for their valuable input. Thanks are also extended patients who participated in this clinical study

\section{References}

Abdel-Bary, EH, Mangoud, AM, El-Hady, HA, Salama, MF, Morsy, TA, 2012: Impact of fibrosis on response to interferon therapy in Egyptian HCV patients. J. Egypt. Soc. Parasitol., V. 42, No. 3, Pp.: 665 - 674

Adaninggar, PN, Ulfa, K, Ummi, M, Poernomo, BS, Swan, AN, et al, 2016: Response evaluation of patients undergoing transarterial chemoembolization (TACE) for HCC with respect to tumor size, number of lesion, and alpha-fetoprotein (AFP) level. Indons. J. Gastroenterol. Hepatol. Digest. Endosco. 5, 3:58-64.

Choi, JY, Jung, SW, Kim, HY, Kim, M, Kim, Y, et al, 2013: Diagnostic value of AFP-L3 and PIVKA-II in hepatocellular carcinoma according to total-AFP. World J Gastroenterol. 19, 3:33946

Darnell, A, Forner, A, Rimola, J, Reig, M, García, Á, et al, 2015: Liver imaging reporting and data system with MR imaging: evaluation in nodules $20 \mathrm{~mm}$ or smaller detected in cirrhosis 
at screening US. Radiol. 275, 3:698-707.

El-Tayeh, SF, Hussein, TD, El-Houseini, ME, Amer, MA, El-Sherbini, M, et al, 2012: Serological biomarkers of hepatocellular carcinoma in Egyptian patients. Dis. Markers 32, 4:255-63.

Faria, SC, Sagebiel, T, Patnana, M, Cox, V, Viswanathan, C, et al, 2019: Tumor markers: myths and facts unfolded. Abdom. Radiol. (NY). 44, 4:1575-600

Gan, Y, Liang, Q, Song, X, 2013: Diagnostic value of alpha-L-fucosidase for hepatocellular carcinoma: a meta-analysis. Tumor Biol. 5, 6: 95-102.

Ibrahim, AM, Hashem, ME, Mostafa, EF, Refaey, MM, Hamed, EF, et al, 2013: Annexin A2 versus Afp as an efficient diagnostic serum marker for hepatocellular carcinoma. J. Gastroenterol. Hepatol. Res. 2, 9:780-5.

Malaguarnera, G, Giordano, M, Paladina, I, Berretta, M, et al, 2010: Serum markers of hepatocellular carcinoma. Springer1 1, 2:10-6.

Montaser, MF, Sakr, MA, Khalifa, MO, 2012: Alpha-L-fucosidase as a tumour marker of hepatocellular carcinoma. Arab J. Gastroenterol. 5, 4:14-30.

Mossad, NA, Mahmoud, EH, Osman, EA, Mahmoud, SH, Shousha, HI, 2014: Evaluation of squamous cell carcinoma antigen-immunoglobulin M complex (SCCA-IGM) \& alpha-L-fucosidase (AFU) as novel diagnostic biomarkers for hepatocellular carcinoma. Tumor Biol. 35, 11:11559-64.

Negahdary, M, Eftekhari, A, Mirzaei, S, Basi- rizadeh, M, Ghobadzadeh, S, 2015: Tumor markers and hepatocellular carcinoma. J. Biol. Today's World 4, 6:124-31.

Reig, M, Darnell, A, Forner, A, Rimola, J, Ayuso, C, et al, 2014: Systemic therapy for hepatocellular carcinoma: Issue of treatment stage migration and registration of progression using the BCLC-refined RECIST. In: Seminars in liver disease, Thieme Medical Publishers.

Saad, ZM, Fouad, Y, Ali, LH, Hassanin, TM, 2020: Clinical significance of annexin $A 4$ as a biomarker in the early diagnosis of HCC. Asian Pac. J. Canc. Prev. 21, 9:2661-5

Siegel, R, Naishadham, D, Jemal, A, 2013: Cancer statistics, 2013. CA: Canc J. Clinicians 63, 1:11-30.

Takahashi, H, Saibara, T, Iwamura, S, Tomita, A, Maeda, T, et al, 1994: Serum $\alpha$-L-fucosidase activity and tumor size in hepatocellular carcinoma hepatology. Hepatology 19, 161: 414-7.

Waidely, E, Al-Youbi, AO, Bashammakh, AS, El-Shahawi, MS, et al, 2017: Alpha-L-fucosidase immunoassay for early detection of hepaticellular carcinoma. Anal. Chem. 89, 17: 9459-66. Wang, JJ, Cao, EH, 2004: Rapid kinetic rate assay of serum a-L-fucosidase in patients with hepatocellular carcinoma by using a novel substrate. Clin. Chim. Acta11; 347-357.

Zhao, YJ, Ju, Q, Li, GC, 2013: Tumor markers for hepatocellular carcinoma. Mol. Clin. Oncol. 1:593-8.

\section{Explanation of figures}

Fig. 1: ROC curve showed both sera AFP \&AFU in differentiating HCC from LC

Fig. 2: ROC curve showed basal and after intervention AFU in prediction of $\mathrm{HCC}$ recurrence.

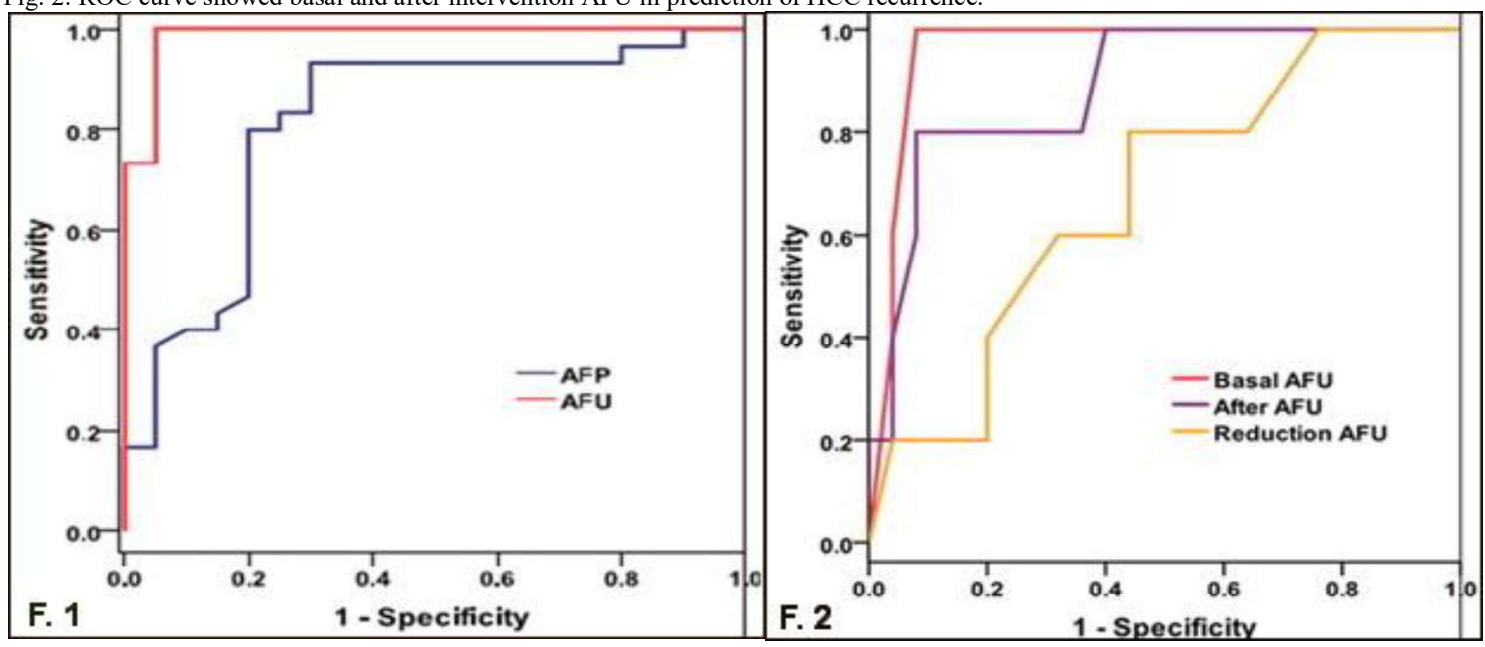

